\title{
SCHUBERT VARIETIES ARE LOG FANO OVER THE INTEGERS
}

\author{
DAVE ANDERSON AND ALAN STAPLEDON
}

\begin{abstract}
Given a Schubert variety $X_{w}$, we exhibit a divisor $\Delta$, defined over $\mathbb{Z}$, such that the pair $\left(X_{w}, \Delta\right)$ is $\log$ Fano in all characteristics.
\end{abstract}

Let $X_{w}=\overline{B w B / B}$ be a Schubert variety in a Kac-Moody flag variety $G / B$ over an algebraically closed field of arbitrary characteristic. Fix a reduced word for the Weyl group element $w$, and let $\varphi: \widetilde{X}_{w} \rightarrow X_{w}$ be the corresponding Bott-Samelson resolution. Let $\partial=\partial_{1}+\cdots+\partial_{k}$ be the complement of the open $B$-orbit in $X_{w}$, written as a sum of prime divisors, and define $\widetilde{\partial}=\widetilde{\partial}_{1}+\cdots+\widetilde{\partial}_{\ell}$ similarly; the divisor $\widetilde{\partial}$ is a simple normal crossings divisor. Here $\ell=\ell(w)$ is equal to the length of $w$, which in turn is equal to the dimension of $X_{w}$.

Denote by $\rho$ the sum of all fundamental weights of (the simply connected form of) $G$, and let $\mathcal{L}(\rho)$ be the corresponding ample line bundle on $X_{w}$. Choosing $B$-invariant sections of $\mathcal{L}(\rho)$ and $\varphi^{*} \mathcal{L}(\rho)$, these line bundles correspond to divisors

$$
a_{1} \partial_{1}+\cdots+a_{k} \partial_{k}, \quad \text { and } \quad b_{1} \widetilde{\partial}_{1}+\cdots+b_{\ell} \widetilde{\partial}_{\ell},
$$

for some nonnegative integers $a_{i}$ and $b_{i}$. In fact, they are all positive, by the Lemma below.

Recall that for a normal irreducible variety $Y$ and an effective $\mathbb{Q}$-divisor $D$, the pair $(Y, D)$ is Kawamata log terminal (klt) if $K_{Y}+D$ is $\mathbb{Q}$-Cartier, and for all proper birational maps $\pi: Y^{\prime} \rightarrow Y$, the pullback $\pi^{*}\left(K_{Y}+D\right)=K_{Y^{\prime}}+D^{\prime}$ has $\pi_{*} K_{Y^{\prime}}=K_{Y}$ and $\left\lfloor D^{\prime}\right\rfloor \leq 0$. The pair is $\log$ Fano if it is klt and $-\left(K_{Y}+D\right)$ is ample.

In [6], Schwede and Smith prove that globally F-regular varieties are log Fano, for some choice of boundary divisor that may depend on the characteristic. Motivated by the fact that Schubert varieties are known to be globally $F$-regular [3], they ask if there exists a single boundary divisor that works uniformly, in all characteristics. The purpose of this note is to answer their question affirmatively:

Theorem. The pair $\left(X_{w}, \Delta\right)$ is log Fano, where $\Delta=c_{1} \partial_{1}+\cdots+c_{k} \partial_{k}$, and $c_{i}=$ $1-a_{i} / M$ for some integer $M$ greater than all $a_{i}$.

We will need a lemma.

Lemma. For all $i$, we have $b_{i}>0$.

Date: March 26, 2012.

2000 Mathematics Subject Classification. 14M15; 14E30; 20 G99.

DA was partially supported by NSF Grant DMS-0902967. 
Proof. Let $\left(\alpha_{1}, \ldots, \alpha_{\ell}\right)$ be the sequence of simple roots corresponding to the reduced word defining $\widetilde{X}_{w}$, and let $s_{1}, \ldots, s_{\ell}$ be the corresponding simple reflections. For each $i$, set

$$
v_{i}=s_{1} \cdots \widehat{s}_{i} \cdots s_{\ell}=w s_{\gamma_{i}}
$$

where

$$
\gamma_{i}=s_{\ell} s_{\ell-1} \cdots s_{i+1}\left(\alpha_{i}\right)
$$

We claim that

$$
b_{i}=\left\langle\rho, \gamma_{i}^{\vee}\right\rangle=\operatorname{ht}\left(\gamma_{i}\right),
$$

where $\operatorname{ht}(\beta)=\left|\sum n_{i}\right|$ if the $\operatorname{root} \beta=\sum n_{i} \alpha_{i}$ as a sum of simple roots. (Since the expression $w=s_{1} \cdots s_{\ell}$ is reduced, each $\gamma_{i}$ is a positive root.)

The claimed expression follows from Chevalley's formula for multiplying a Schubert class by a divisor [1], but it is easy enough to prove directly. We first review some basic geometry of the Bott-Samelson variety. We have $\widetilde{X}_{w}=\left(P_{\alpha_{1}} \times \cdots \times\right.$ $\left.P_{\alpha_{\ell}}\right) / B^{\ell}$, where $P_{\alpha}$ is the minimal parabolic subgroup, and $B^{\ell}$ acts by

$$
\left(p_{1}, p_{2}, \ldots, p_{\ell}\right) \cdot\left(b_{1}, b_{2}, \ldots, b_{\ell}\right)=\left(p_{1} b_{1}, b_{1}^{-1} p_{2} b_{2}, \ldots, b_{\ell-1}^{-1} p_{\ell} b_{\ell}\right) .
$$

For each $i$, fix a representative $\dot{s}_{i} \in P_{\alpha_{i}}$. The torus $T$ acts on $\widetilde{X}_{w}$ by left multiplication, and its fixed points correspond to subsets $I \subseteq[\ell]:=\{1, \ldots, \ell\}$. Specifically, the point $p(I)$ is defined by $p_{i}=\dot{s}_{i}$ if $i \in I$ and $p_{i}=e$ otherwise. The divisor $\widetilde{\partial}_{i}$ is defined by the equation $p_{i}=e$.

For each $i=1, \ldots, \ell$, one can find a $T$-invariant curve $C_{i}$ in $\widetilde{X}_{w}$, whose fixed points are $p([\ell])$ and $p([\ell] \backslash\{i\})$. By considering $T$-fixed points, it is easy to see that $C_{i} \cap \widetilde{\partial}_{i}=p([\ell] \backslash\{i\})$, and $C_{i} \cap \widetilde{\partial}_{j}=\emptyset$ if $i \neq j$. It follows that $b_{i}=\left(\varphi^{*} \mathcal{L}(\rho) \cdot C_{i}\right)$.

By the projection formula, this intersection number is equal to $\left(\mathcal{L}(\rho) \cdot \varphi\left(C_{i}\right)\right)$. The curve $\varphi\left(C_{i}\right)$ is the unique $T$-invariant curve in $X_{w} \subseteq G / B$ with fixed points $w B$ and $v_{i} B$. The line bundle $\mathcal{L}(\rho)$ on $G / B$ is invariant under the action of the Weyl group, so we may apply a translation by $w^{-1}$; now $w^{-1} \varphi\left(C_{i}\right)$ is the curve with fixed points $e B$ and $w^{-1} v_{i} B=s_{\gamma_{i}} B$. The formula (3) follows, since for any dominant weight $\lambda$ and any positive root $\beta$, if $C$ is the $T$-invariant curve in $G / B$ joining $e B$ and $s_{\beta} B$, we have $(\mathcal{L}(\lambda) \cdot C)=\left\langle\lambda, \beta^{\vee}\right\rangle$.

Proof of Theorem. The canonical divisors for $X_{w}$ and $\widetilde{X}_{w}$ are well known (see, e.g., $[5])$. We have

$$
K_{X_{w}}=-\left(a_{1}+1\right) \partial_{1}-\cdots-\left(a_{k}+1\right) \partial_{k}
$$

and

$$
K_{\widetilde{X}_{w}}=-\left(b_{1}+1\right) \widetilde{\partial}_{1}-\cdots-\left(b_{\ell}+1\right) \widetilde{\partial}_{\ell} .
$$

By the definition of $\Delta$, the (integral) divisor

$$
\begin{aligned}
M K_{X_{w}}+M \Delta & =\left(-M a_{1}-M+M-a_{1}\right) \partial_{1}+\cdots+\left(-M a_{1}-M+M-a_{1}\right) \partial_{k} \\
& =-(M+1) a_{1} \partial_{1}-\cdots-(M+1) a_{k} \partial_{k}
\end{aligned}
$$


comes from a section of $\mathcal{L}(-\rho)^{\otimes M+1}$, so $K_{X_{w}}+\Delta$ is $\mathbb{Q}$-Cartier and anti-ample.

Now set $\widetilde{c}_{i}=1-b_{i} / M$, and let $\widetilde{\Delta}=\sum \widetilde{c}_{i} \widetilde{\partial}_{i}$. From the definition, we have

$$
\begin{aligned}
\varphi^{*}\left(K_{X_{w}}+\Delta\right) & =\frac{M+1}{M}\left(-b_{1} \widetilde{\partial}_{1}-\cdots-b_{\ell} \widetilde{\partial}_{\ell}\right) \\
& =\left(-b_{1}-1+\frac{1}{M}\left(M-b_{1}\right)\right) \widetilde{\partial}_{1}+\cdots+\left(-b_{\ell}-1+\frac{1}{M}\left(M-b_{\ell}\right)\right) \widetilde{\partial}_{\ell} \\
& =K_{\widetilde{X}_{w}}+\widetilde{\Delta} .
\end{aligned}
$$

The Lemma implies that $\lfloor\widetilde{\Delta}\rfloor \leq 0$.

Next we show that $\varphi_{*} \widetilde{\Delta}=\overline{\widetilde{\Delta}}$. Since $X_{w}$ is normal, for each $j=1, \ldots, k$, there is a unique $i(j)$ such that $\varphi\left(\widetilde{\partial}_{i(j)}\right)=\partial_{j}$; the remaining $\widetilde{\partial}_{i}$ 's are collapsed by $\varphi$. It follows that for any divisor $\widetilde{D}=\sum_{i} d_{i} \widetilde{\partial}_{i}$, we have $\varphi_{*} D=\sum_{j} d_{i(j)} \partial_{j}$. For the same reason, $\varphi_{*} \varphi^{*} \mathcal{L}(\rho)=\mathcal{L}(\rho)$, so $a_{j}=b_{i(j)}$. The claim follows.

Finally, the map $\varphi: \widetilde{X}_{w} \rightarrow X_{w}$ is a rational resolution (see [5]), so in particular we have $\varphi_{*} K_{\widetilde{X}_{w}}=K_{X_{w}}$. Applying [2, Lemma 2.30 and Corollary 2.31], it follows that $\left(X_{w}, \Delta\right)$ is klt, and the Theorem is proved.

Remark. The formula

$$
-K_{\widetilde{X}_{w}}=\sum_{i}\left(\left\langle\rho, \gamma_{i}^{\vee}\right\rangle+1\right) \widetilde{\partial}_{i}
$$

proved in the Lemma can be found in [4, Proof of Proposition 10], and is also valid for Bott-Samelson varieties corresponding to non-reduced words. In this generality the coefficients on the right-hand side may be negative, since some of the $\gamma_{i}$ 's will be negative roots. Note, however, that the anticanonical divisor is always effective.

Acknowledgements. We thank Karl Schwede and Karen Smith for asking us this question, as well as Shinnosuke Okawa and the referee for helpful comments.

\section{REFERENCES}

[1] C. Chevalley, "Sur les décompositions cellulaires des espaces G/B," Proc. Sympos. Pure Math. 56 1-23, Amer. Math. Soc., Providence, 1994.

[2] J. Kollár and S. Mori, Birational Geometry of Algebraic Varieties, Cambridge, 1998.

[3] N. Lauritzen, U. Raben-Pedersen, and J. F. Thomsen, "Global F-regularity of Schubert varieties with applications to D-modules," J. Amer. Math. Soc. 19 (2006), no. 2, 345-355.

[4] V. B. Mehta and A. Ramanathan, "Frobenius splitting and cohomology vanishing for Schubert varieties," Ann. of Math. 122 (1985), no. 1, 27-40.

[5] A. Ramanathan, "Schubert varieties are arithmetically Cohen-Macaulay," Invent. Math. 80 (1985), 283-294.

[6] K. Schwede and K. E. Smith, "Globally F-regular and log Fano varieties," Adv. Math. 224 (2010), no. 3, 863-894.

Department of Mathematics, University of Washington, Seattle, WA 98195

Department of Mathematics, University of British Columbia, BC, Canada V6T $1 Z 2$

E-mail address: anderson@math.ubc.ca, dandersn@math.washington.edu

E-mail address: astapldn@math.ubc.ca 